\title{
Uma estratégia baseada em medida de centralidade para instalação de pontos de disseminação em VANETs
}

\author{
Douglas L. L. Moura ${ }^{1}$, Raquel S. Cabral ${ }^{1}$,Thiago B. M. Sales ${ }^{1}$, André L. L. Aquino ${ }^{2}$ \\ ${ }^{1}$ Curso de Ciência da Computação - Universidade Federal de Alagoas (UFAL) \\ Caixa Postal 57.309-005 - Arapiraca - AL - Brasil \\ ${ }^{2}$ Instituto de Computação - Universidade Federal de Alagoas (UFAL) \\ Caixa Postal 57.072-970 - Maceió - AL - Brasil \\ \{douglas.moura, thiago.sales, raquel.cabral\}earapiraca.ufal.br, \\ alla@laccan.ufal.br
}

\begin{abstract}
The vehicular networks present a high dynamic topology that requires an infrastructure of fixed transmission points installed along the roads, which may provide vehicle connectivity in sparse or low density areas. This work presents an approach based on genetic algorithm and centrality measure to Maximum Coverage with Time Threshold Problem (MCTTP) in vehicular networks. Our approach considers a limited number of dissemination points to maximize vehicle coverage that allows vehicle connectivity. The centrality measure from the complex network theory reduces the problem's search space of the genetic algorithm, which implies to a more efficient approach. We applied our proposed solution to a real urban scenario with a high vehicles density. Experimental results suggest a higher vehicle coverage of $1.06 \%$ and $3.78 \%$ than the results of other approaches.
\end{abstract}

Resumo. As redes veiculares possuem uma topologia intrinsecamente dinâmica, tornando-se necessária a instalação de pontos de disseminação como complemento à comunicação realizada apenas entre os veículos. Neste trabalho apresentamos uma abordagem baseada em algoritmos genéticos e medidas de centralidade para o problema da máxima cobertura com tempo de contato (MCTTP) em redes veiculares. A abordagem considera a instalação de um número limitado de pontos de disseminação para permitir a propagação de informação maximizando a cobertura de veículos. A utilização da medida de centralidade permitiu a redução do espaço de busca do algoritmo genético, tornando-o mais eficiente. A solução foi aplicada a um cenário urbano real com uma alta densidade de veículos que se deslocam ao longo do tempo. Os resultados obtidos foram comparados com outras duas abordagens da literatura. Considerando um tempo mínimo de contato, os resultados encontrados sugerem um aumento significativo na cobertura dos veículos, $1.06 \%$ e $3.78 \%$ quando comparados aos resultados obtidos por outras soluções.

\section{Introdução}

Em uma VANET (Vehicular Ad hoc Network) [Al-Sultan et al. 2014], os veículos são capazes de trocar mensagens com outros veículos (comunicação V2V - Vehicle-to-Vehicle) 
ou com pontos de disseminação (comunicação V2I - Vehicle-to-Infrastructure) que são instalados ao longo das estradas. Em razão do alto dinamismo nos padrões de mobilidade da comunicação V2V, a topologia da rede é constantemente alterada, o que implica em uma conectividade frágil e desconexões. Por outro lado, apesar dos custos associados à adoção dos pontos de disseminação (PDs), a comunicação V2I permite reduzir os limites na propagação de dados. Desta forma, os PDs podem promover a disseminação escalável de informações ao longo das estradas.

Dessa forma, é possível definir o seguinte problema:

dado um conjunto $K$ de pontos de disseminação, onde implantar os elementos de $K$ de modo a maximizar o contato entre veículos e pontos de disseminação, bem como garantir um tempo mínimo na cobertura para conexões entre veículos e pontos de disseminação?

O problema de cobertura e conectividade trata-se de um desafio clássico em redes ad hoc no geral [Meguerdichian et al. 2001], nas quais a cobertura é uma métrica de qualidade de comunicação. Apesar de existirem soluções difundidas na literatura que são interessantes e eficientes, em razão das particularidades da VANET, estratégias diferenciadas são importantes como forma de lidar com o comportamento dinâmico da rede veicular. O problema de MCTTP é abordado em [Trullols et al. 2010] e [Cavalcante et al. 2012], onde são apresentados uma solução gulosa e um algoritmo genético para o problema, respectivamente.

Neste trabalho apresentamos uma solução baseada em algoritmos genéticos e uma medida de centralidade, denominada betweenness centrality [Brandes 2001], para o problema de MCTTP. Além disso, foi utilizado o método de Louvain [Blondel et al. 2008] para detecção de comunidades, com o objetivo de reduzir o espaço de busca do problema e tornar a solução mais eficiente. Considerando um tempo mínimo de contato, os resultados encontrados sugerem um aumento significativo na cobertura dos veículos, de até $1.06 \%$ e $3.78 \%$ quando comparados aos resultados obtidos por [Cavalcante et al. 2012] e [Trullols et al. 2010].

O restante deste artigo está organizado da seguinte forma: Seção 2 apresenta uma revisão bibliográfica sobre o problema tratado. Seção 3 descreve o problema de máxima cobertura com tempo de contato em redes veiculares, apresentando a formalização do problema. Seção 4 detalha a solução baseada na métrica de centralidade, ao passo que são discutidos na Seção 5 os resultados encontrados com a abordagem proposta, comparandoa a duas abordagens encontradas na literatura. Por fim, Seção 6 mostra a conclusão e alguns trabalhos futuros.

\section{Trabalhos Relacionados}

Nesta seção são apresentados os principais trabalhos relacionados a implantação de pontos de disseminação ao longo de uma topologia rodoviária. Inicialmente, temos que Aslam et al. [Aslam et al. 2012] propuseram dois métodos de otimização para determinar em quais interseções instalar um número limitado de PDs, com o objetivo de maximizar o fluxo de informações dos veículos para os PDs. O primeiro método, baseado na Programação Inteira Binária (PIB), utilizou Branch-and-Bound (B\&B) para encontrar a solução ótima. O segundo método utiliza a heurística da expansão do balão (Balloon Expansion Heuristic - BEH) e encontrou a solução ótima incorporando o conhecimento 
da topologia das estradas. Dentre os métodos propostos, como esperado, o segundo destacou-se em razão de seu tempo de execução, que é consideravelmente menor que o primeiro método.

Brahim et al. [Ben Brahim et al. 2015] propuseram soluções nas quais o objetivo era melhorar o desempenho, confiabilidade e conectividade da rede. A rede foi representada como um grafo ponderado, de maneira que o peso atribuído a cada aresta estava relacionado a importância de sua ligação. Desta forma, os autores resolveram o problema de instalação dos PDs utilizando duas abordagens: problema da mochila; e algoritmo de PageRank. Nesse contexto, o problema da mochila obteve melhores resultados.

Por outro lado, considerando a disseminação de informações em VANETs, alguns autores buscaram modelar o problema a partir do Problema da Máxima Cobertura (Maximum Coverage Problem - MCP) ou variantes do MCP [Silva et al. 2015]. Trullols et al. [Trullols et al. 2010] propuseram duas outras abordagens baseadas no problema da mochila e no problema de máxima cobertura com tempo de contato (MCTTP). Em cada solução, os autores aplicaram o algoritmo guloso e a estratégia dividir para conquistar. Baseando-se nesses resultados, Cavalcante et al. [Cavalcante et al. 2012] considerou o mesmo problema e usou como solução um algoritmo genético. A partir da inclusão da informação gerada pelo algoritmo guloso, foi possível melhorar a solução do algoritmo genético e obter um aumento no percentual de cobertura.

Outras abordagens são propostas na literatura com diferentes estratégias de solução. Por exemplo, modelos probabilísticos [Chi et al. 2016, Silva et al. 2014], modelos que utilizam grafos para caracterizar o padrão de mobilidade dos veículos [Xiong et al. 2013] e soluções baseadas na Programação Linear Inteira (PLI) [Liang et al. 2012, Cumbal et al. 2016]. Todos os trabalhos mencionados utilizaram cenários específicos e parâmetros pré-definidos.

Neste trabalho, o problema foi modelado como um problema de máxima cobertura com tempo de contato utilizando uma abordagem genética adicionando o uso de uma medida de centralidade para otimizar o espaço de busca do algoritmo. Dessa forma foi possível obter um algoritmo mais eficiente em relação à porcentagem de cobertura de veículos, ao número de gerações do algoritmo genético para encontrar a solução e, consequentemente, ao tempo de execução.

\section{Definição do Problema de Otimização}

Partimos da definição de que um veículo será coberto se, e somente se, estiver conectado a um ou mais PDs por um certo período de tempo. O problema foi modelado como um problema de máxima cobertura (MCTTP) [Hochbaum 1997]. O MCTTP é um problema de otimização combinatória NP-Difícil [Khuller et al. 1999]. Assim, torna-se necessário o uso de abordagens heurísticas como forma de obter uma solução aproximada para o problema.

Inicialmente, seja $\mathbf{V}=\left\{V_{1}, V_{2}, \ldots, V_{n}\right\}$ o conjunto de veículos presentes na topologia rodoviária e $n$ o número máximo de veículos; $\mathbf{I}=\left\{I_{1}, I_{2}, \ldots, I_{m}\right\}$ o número de interseções presentes na topologia; $\widehat{\mathbf{I}}$ o conjunto de interseções escolhidas para a instalação dos PDs, i.e., a lista com as posições dos PDs; e $T[m \times n]$ uma matriz que indica a relação entre os veículos e interseções, em que cada elemento $T_{i j}$ da matriz é o 
tempo total de contato entre o veículo $j$ e a interseção $i$, i.e., as ligações V2I. O conjunto de cobertura $\mathbf{V}^{\prime}=\left\{V_{I_{1}}^{\prime}, V_{I_{2}}^{\prime}, \ldots, V_{I_{m}}^{\prime}\right\}$ é determinado por todos os veículos que atravessam alguma interseção, onde $V_{I_{i}}^{\prime} \in \mathbf{V}^{\prime}$ é o subconjunto de veículos presentes na interseção $I_{i}$. Um dado veículo é considerado coberto quando permanecer em contato com algum PD por no mínimo $\tau$ segundos. A cobertura pode ser completada de forma direta (único PD é suficiente para atingir $\tau$ ) ou fragmentada (o tempo de cobertura é alcançado com outros PDs que o veículo esteve em contato).

Desta maneira, o MCTTP seleciona $k$ conjuntos de $\mathrm{V}^{\prime}$ para cobrir a maior quantidade possível de veículos no cenário com um tempo mínimo de $\tau$ segundos. O problema de máxima cobertura com tempo de contato pode ser formulado como segue:

$$
\max \sum_{j=1}^{n}\left[\min \left(\tau, \sum_{i=1}^{m} T_{i j} \mathcal{Y}_{i}\right)\right] \text {, }
$$

sujeito a

$$
\sum_{i=1}^{m} \mathcal{Y}_{i} \leq k ; \quad \mathcal{Y}_{i} \in\{0,1\} \forall i
$$

Na Equação 1 é descrito o MCTTP, onde $\mathcal{Y}_{i}$ é 1 se o PD está na interseção $i$, de forma que se maximize o número de veículos cobertos. A restrição 2 assegura que o número de PDs seja menor ou igual a $k$ interseções.

\section{Solução proposta}

A nossa solução considera o fluxo presente na Figura 1, cujo as etapas são descritas como segue:

Figura 1. Fases da solução proposta

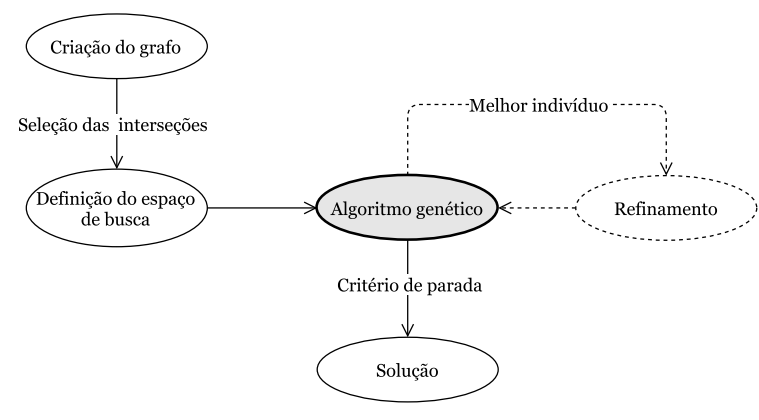

O ponto inicial da solução é a criação do grafo bipartido $G=(\mathcal{V}, \mathcal{E})$ nãodirecionado e ponderado, em que $\mathcal{V}=\mathbf{V} \cup \mathbf{I}$ e $\mathcal{E}_{l}=\left(\mathbf{V}_{j}, \mathbf{I}_{i}\right)$, em que o par ordenado $\left(\mathbf{V}_{j}, \mathbf{I}_{i}\right)$ é um elemento da matriz $\mathbf{T}[m \times n]$. Cada veículo e interseção recebem um valor para identificar cada um deles no grafo. $\mathrm{O}$ valor $\mathbf{T}_{i j}$ associado ao tempo de cobertura deve ser maior que 0 . Dado que o grafo é não-direcionado, se um veículo $j$ está no raio de transmissão de um PD na interseção $i$, então este PD também estará no raio de transmissão do veículo $j$. Em redes reais, em razão das diferenças nas potências dos sinais, 
tal simetria nem sempre ocorre. Ademais, vértices e interseções isolados são retirados do grafo. O peso da conexão entre a interseção $i$ e o veículo $j$ é dado por:

$$
p_{i j}=\max (\mathbf{T})-\min \left(\tau, \mathbf{T}_{i j}\right)+1
$$

Assim, o peso é obtido a partir da diferença entre o maior tempo de contato na matriz $\max (\mathbf{T})$, e o menor valor $\tau$ e o elemento $\mathbf{T}_{i j}$. Por fim, o valor 1 é somado ao resultado para evitar a ocorrência de uma aresta com peso nulo.

O espaço de busca denota todas as possíveis soluções para o problema tratado. Diante disso, partindo de uma população inicial, o algoritmo genético irá explorar diversas regiões na tentativa de alcançar o máximo global, nesse caso, o conjunto de interseções onde deve-se instalar os PDs de forma a garantir a maior cobertura de veículos. Neste trabalho, a definição do espaço de busca é feita em duas etapas: (i) o uso de uma medida de centralidade, para que as interseções mais relevantes sejam escolhidas para a instalação dos PDs, i.e., este procedimento busca identificar interseções que tendem a favorecer a cobertura dos veículos devido a sua centralidade; (ii) e a detecção de comunidades devido à alta densidade da rede. A seleção das interseções candidatas tem como objetivo reduzir o espaço de busca do problema, i.e., é um pré-processamento da entrada do algoritmo genético.

O betweenness centrality é uma medida que quantifica a centralidade de um vértice com base no número de caminhos mínimos que passam por este vértice e é dado pela Equação 4 [Newman 2004]:

$$
B_{v}=\sum_{i \neq v \neq j \in V} \frac{\sigma_{i j(v)}}{\sigma_{i j}}
$$

Considere $\sigma_{i j(v)}$ como sendo a quantidade de caminhos mínimos entre os vértices $i$ e $j$ que passam pelo vértice $v$, e $\sigma_{i j}$ é o número total de caminhos mínimos entre os vértices $i$ e $j$. Assim, quanto maior o betweennes de um vértice, maior sua importância dentro da rede.

A partir do grafo $G=(\mathcal{V}, \mathcal{E})$, são identificados um conjunto de comunidades $\mathcal{C}=\left\{C_{1}, C_{2}, \ldots, C_{c}\right\}$ em que cada elemento de $\mathcal{C}$ é subgrafo de $G$, i.e., $C_{i}=\left(\mathcal{V}^{\prime}, \mathcal{E}^{\prime}\right)$, onde $\mathcal{V}^{\prime} \subseteq V$ e $\mathcal{E}^{\prime} \subseteq \mathcal{E}$. Para a detecção das comunidades é utilizado o método heurístico de Louvain baseado na otimização de modularidade e proposto como solução para o problema de detecção de comunidades em redes de grande escala [Fortunato 2010]. Após formadas as comunidades, o betweenness centrality é calculado para todas as interseções (vértices) de cada comunidade do conjunto $\mathcal{C}$. Posteriormente, cada comunidade é percorrida e a interseção com maior valor de betweenness centrality é selecionada. Processo é repetido até selecionar um percentual de $q i$ interseções, valor de $q i$ é definido durante o ajuste dos parâmetros do algoritmo.

Visto que a medida de centralidade é atribuída a cada vértice da comunidade $C_{c}$, o resultado será um conjunto Q contendo o valor de centralidade de cada interseção e sua respectiva comunidade.

O algoritmo genético (ver Algoritmo 1), denominado de MCTTP-c, é baseado no betweenness centrality. A entrada do algoritmo é o número $k$ de PDs que serão instalados, a matriz $\mathbf{T}$, o tempo de contato $\tau$, o conjunto $\mathbf{V}^{\prime}$ de veículos que tiveram contato 
com alguma interseção e o conjunto Q. A população inicial do algoritmo é gerada aleatoriamente, contudo, restrita às interseções contidas em Q (Linha 1). Um indivíduo (IN) representa uma possível solução para o problema, com $k$ interseções para implantar $k$ PDs. Assim a função fitness tem como resultado a porcentagem de veículos cobertos pelos PDs. A função fitness $\mathcal{F}_{I N}$ é definida pela Equação 5:

$$
\mathcal{F}_{I N}=\frac{|\widehat{V}|}{n}
$$

Em que $n$ é o total de veículos e $\widehat{V}$ é o conjunto dos veículos cobertos pelos PDs instalados nas interseções pertencentes ao indivíduo IN. Cada elemento $\widehat{V}$ deve possuir um tempo de contato maior que $\tau$.

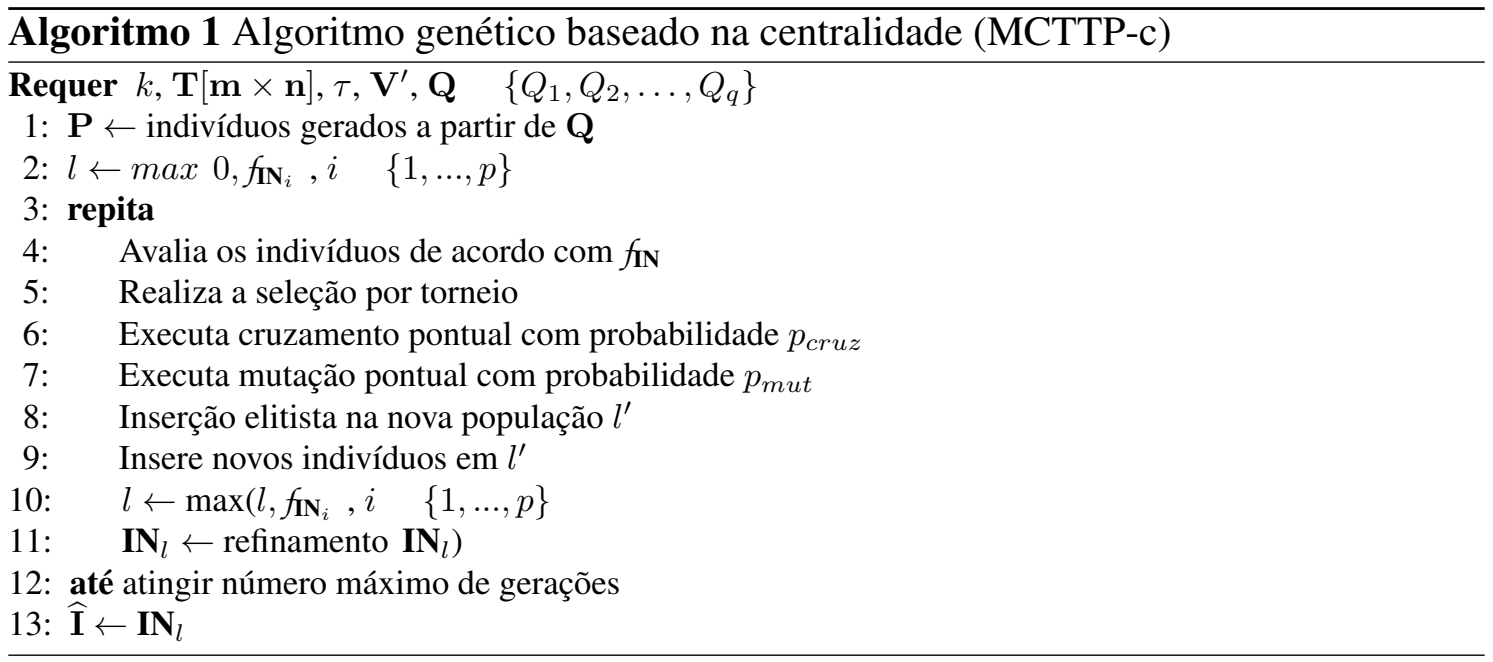

Após a inicialização da população, os indivíduos têm a sua aptidão avaliada (Linha 2) e durante um número mínimo de gerações são submetidos aos processos de seleção natural. Desta forma, os indivíduos são selecionados através de torneio e são submetidos ao cruzamento pontual para compor a nova população (Linhas 5 e 6). Os novos indivíduos podem sofrer mutação pontual (Linha 7) e o elitismo preserva o indivíduo mais apto para compor a população seguinte (Linha 8). Os indivíduos são novamente avaliados (Linha 10) e o melhor indivíduo é então submetido ao processo de refinamento (Linha 11). Ao atingir o critério de parada (Linha 12), o algoritmo irá retornar o melhor indivíduo da população $\widehat{\mathbf{I}}$.

O processo de refinamento consiste em melhorar a aptidão do indivíduo $\mathbf{I N}_{l}$ a partir da troca de um gene, escolhido aleatoriamente, por outro que esteja na mesma comunidade. O gene substituto é aquele cuja troca resultar no maior valor de aptidão para o indivíduo. O método de refinamento é aplicado após a seleção das interseções candidatas, em razão disso, apenas as interseções mais relevantes da comunidade explorada serão consideradas. Por fim, tem-se a solução do algoritmo com os PDs instalados garantindo a maior cobertura de veículos.

\section{Experimentos e resultados}

Os experimentos foram executados a partir de um cenário elaborado com dados extraídos de traços de mobilidade veicular, que descrevem a posição individual dos veículos durante 
um período de observação. O raio de transmissão assumido para cada nó da rede foi de $R=100$ metros, valor tipicamente adotado em experimentos. Questões relativas a protocolos de rede e roteamento foram abstraídas, assim como eventuais problemas que poderiam resultar na falha da entrega das mensagens, por exemplo, atenuação de sinal. Deste modo, o tempo de permanência de um veículo em uma interseção qualquer foi computado a partir da Distância Euclidiana.

O cenário foi construído, a partir de dados extraídos dos traços de mobilidade da área urbana da cidade Colônia, na Alemanha ${ }^{1}$. O conjunto original de dados cobre uma área de $400 \mathrm{~km}^{2}$ por um período de 24 horas, mais de 700.000 rotas individuais de veículos foram utilizadas. Desta base de $24 \mathrm{~h}$, dado a magnitude dos dados e o custo computacional para processá-los, utilizamos apenas um conjunto de dados reduzido ${ }^{2}$ contendo um intervalo de duas horas de simulação. Os dados extraídos reproduzem o cenário em um horário de pico de um dia matinal de trabalho ( $6 \mathrm{~h}$ às $8 \mathrm{~h}$ ), seguindo uma granularidade de um segundo. Este cenário reduzido foi filtrado de modo a remover veículos que atravessaram menos de três interseções ou permaneceram no cenário por um tempo inferior à um minuto. Assim, para construção da matriz de contatos $\mathbf{T}[m \times n]$, contamos com 27,615 interseções e 118,482 veículos.

Os experimentos analisam o percentual de veículos cobertos a partir da solução desenvolvida neste trabalho (MCTTP-c) em comparação com outras duas abordagens, o algoritmo guloso (MCTTP-g) proposto por [Trullols et al. 2010] e o algoritmo genético (MCTTP-cv) de [Cavalcante et al. 2012]. A configuração dos parâmetros influencia no comportamento dos algoritmos genéticos, assim, o ajuste apropriado permite obter melhores resultados. Vários testes foram realizados, permitindo investigar as soluções obtidas para diferentes complexidades do problema e para se chegar aos valores de todos os parâmetros apresentados aqui. Em razão disso, para cada configuração dos algoritmos, estes foram replicados 10 vezes, um número suficiente, considerando o alto custo computacional associado aos experimentos, e o teste Shapiro-Wilk permitiu averiguar a normalidade das distribuições. Adiante, o teste t-Student foi utilizado como critério de decisão do melhor resultado, com intervalo de confiança de $95 \%$ e nível de significância $\alpha$ de $5 \%$. Nos casos em que não se conseguiu determinar a melhor configuração, optou-se por escolher o parâmetro que favorecesse a obtenção da melhor solução considerando um número de gerações menor. Os valores utilizados ao fim do ajuste dos parâmetros dos algoritmos foram os seguintes:

\begin{tabular}{cccccc}
\hline Algoritmo & $q i$ & Gerações & População & Cruzamento & Mutação \\
\hline MCTTP-c & $5 \%$ & 100 & 400 & 0.95 & 0.100 \\
MCTTP-cv & - & 200 & 500 & 0.95 & 0.100 \\
\hline
\end{tabular}

O torneio de tamanho dois foi utilizado em ambas as abordagens.

O parâmetro $k$ foi fixado em 25 , uma quantidade capaz de cobrir um percentual acima de $30 \%$ dos veículos, em razão da dimensão do cenário considerado. Em todos os casos avaliados, o tempo mínimo de contato $\tau$ foi definido como 30 segundos. A Figura 2(a) apresenta um comparativo entre as três abordagens analisadas quando o número

\footnotetext{
${ }^{1} \mathrm{O}$ conjunto de dados é disponibilizado pelo projeto TAPASCologne, uma iniciativa do Institute of Transportation Systems (ITS-DLR) no Centro Aeroespacial Alemão.

${ }^{2}$ Disponível em: http://kolntrace.project.citi-lab.fr/
} 
de PDs é $k=25$. O MCTTP-c obteve um ganho na cobertura dos veículos de $3.05 \%$ (3.600 veículos cobertos) em relação ao MCTTP-g e 0.73\% (864 veículos cobertos) em relação ao MCTTP-cv. É visível que as duas abordagens evolutivas conseguem resultados melhores que a solução gulosa, isto deve-se ao fato de que o algoritmo guloso realiza uma busca local na tentativa de encontrar o máximo global. Em virtude disto, interseções que poderiam complementar a cobertura são desconsideradas por não sugerirem a obtenção de boas soluções. A inserção da restrição de tempo mínimo impõe uma complexidade adicional ao problema, pois ainda que o cenário apresente interseções com um alto tempo de contato, outras devem ser selecionadas a fim de incrementar o tempo de cobertura, que é acumulativo.

Ambos os algoritmos genéticos tratam-se de soluções mais sofisticadas para a resolução do MCTTP, a diferença nos resultados obtidos pelas duas abordagens, apesar de estatisticamente significativa, foi menor, isto sugere uma aproximação do ponto de ótimo global. A sútil diferença entre o MCTTP-cv e o MCTTP-c deve-se aos aprimoramentos aplicados ao MCTTP-c, que permitiu ao algoritmo alcançar regiões melhores do espaço de busca. A partir da seleção de interseções candidatas foi possível excluir regiões de baixa aptidão e direcionar a busca através de regiões mais promissoras. O método de refinamento garantiu ao algoritmo atingir melhores soluções a partir da exploração de interseções que devido à sua similaridade compõe uma mesma comunidade.

Figura 2. Resultados obtidos pelos algoritmos analisados

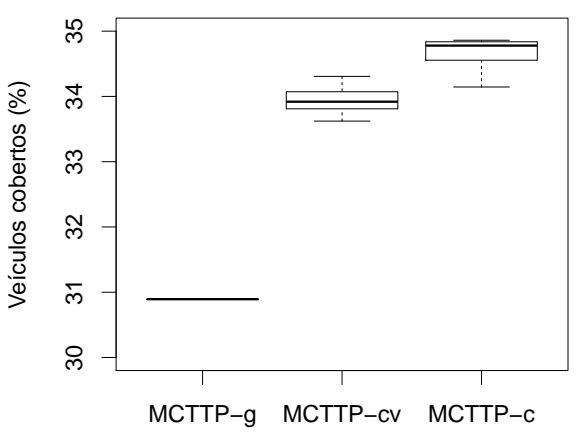

(a) Resultados com $k=25$

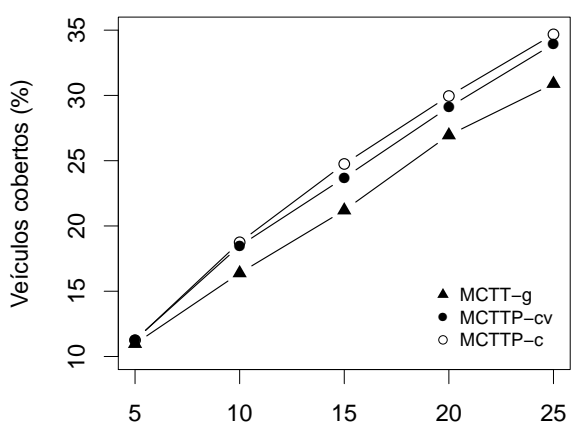

(b) Resultados com variações de $k$

A Figura 2(b) apresenta a evolução dos veículos cobertos para as três abordagens comparadas em função do valor de $k$. Com valores menores para $k$ é notável a dificuldade em encontrar soluções melhores que aquela fornecida pelo MCTTP-g, em $k=5$, por exemplo, os percentuais de cobertura aproximaram-se ao ponto de se sobreporem.

A partir de $k=10$, a diferença entre as três abordagens passa a ser significativa, por conseguinte, na medida em que $k$ assume valores maiores, os percentuais de cobertura obtidos pelas duas abordagens evolutivas claramente superam os resultados do MCTTP-g. Com exceção de $k=5$, que não houve melhora significativa, os percentuais de cobertura alcançados com a solução do MCTTP-c são superiores para todos os valores de $k$. A sútil diferença entre os resultados do MCTTP-c e MCTTP-cv prevalece nos demais casos analisados.

Como forma de analisar a significância estatística de cada caso, o intervalo de 
confiança foi calculado para as soluções baseadas em abordagens evolutivas. A Tabela 1 sintetiza os resultados encontrados para um intervalo de confiança (IC) de 95\%, nível de significância $\alpha$ de $5 \%$, o percentual médio e o percentual da melhor solução obtida para cada implementação do algoritmo genético.

Tabela 1. Variação do número de PDs

\begin{tabular}{rlccccc}
\hline & & 5 & 10 & 15 & 20 & 25 \\
\hline \multirow{3}{*}{ MCTTP-c } & Melhor & 11.28 & 18.75 & 24.77 & 30.22 & 34.86 \\
& Média & 11.25 & 18.75 & $\mathbf{2 4 . 7 5}$ & 29.95 & $\underline{34.67}$ \\
& IC & $(11.20,11.29)$ & $(18.75,18.75)$ & $(24.72,24.78)$ & $(29.84,30.06)$ & $(34.51,34.83)$ \\
\hline \multirow{2}{*}{ MCTTP-cv } & Melhor & 11.28 & 18.62 & 24.10 & 29.63 & 34.30 \\
& Média & 11.28 & 18.47 & $\mathbf{2 3 . 6 9}$ & 29.09 & 33.94 \\
& IC & $(11.28,11.28)$ & $(18.38,18.57)$ & $(23.48,23.90)$ & $(28.89,29.29)$ & $(33.78,34.10)$ \\
\hline MCTTP-g & 10.97 & 16.38 & 21.19 & 26.95 & $\underline{30.89}$ \\
\hline
\end{tabular}

Considerando todos os valores de $k$, o melhor resultado do MCTTP-c em relação ao MCTTP-g foi em $k=25$ (sublinhado), com um ganho na cobertura dos veículos de $3.78 \%$ (4.479 veículos cobertos). Por outro lado, o menor ganho para os algoritmos considerados foi em $k=5$ com um ganho de $0.28 \%$ (331 veículos cobertos) na cobertura dos veículos. Ao comparar as duas abordagens evolutivas, a pequena diferença nos resultados das duas soluções torna-se mais notável. O maior aumento de cobertura obtido pelo MCTTP-c foi em $k=15$ (em negrito), com um ganho de $1.06 \%$ (1.255 veículos cobertos) em relação ao MCTTP-cv.

Dentre os casos mencionados, $k=5$ foi a única ocorrência em que a média do MCTTP-cv foi superior à média do MCTTP-c. Mesmo com uma diferença discreta entre as duas médias, a sobreposição nos intervalos de confiança revela que não existe diferença significativa entre os dois casos, em razão disto, não é possível assumir que os resultados do MCTTP-c sejam inferiores aos do MCTTP-cv. Para os outros casos, indo de acordo com resultados apresentados anteriormente, a solução baseada em centralidade exibe uma média superior às médias do MCTTP-cv e MCTTP-g. Não somente isso, a partir do intervalo de confiança é possível evidenciar que a diferença entre os algoritmos genéticos possui significância estatística para todos os casos a partir de $k=10$.

\section{Conclusão}

Este trabalho apresentou uma solução para a instalação de PDs que foi modelada como o problema da máxima cobertura com tempo de contato, onde um número limitado de PDs deveria maximizar a cobertura dos veículos no cenário por um tempo mínimo de contato. A solução utilizou o betweenness centrality para encontrar interseções com potencial de compor soluções do algoritmo genético. Nossa abordagem, quando comparada com outras duas soluções da literatura, obteve resultados satisfatórios, conseguindo um aumento na cobertura dos veículos de até 3.78\% (4.479 veículos cobertos) em relação ao MCTTP-g. Para o MCTTP-cv, a diferença apesar de ser mais discreta, onde o melhor resultado foi um ganho de $1.06 \%$ (1.255 veículos cobertos), mostra que através de centralidade é possível encontrar soluções que superam aquelas fornecidas pela abordagem evolutiva com informação proveniente da abordagem gulosa. Como trabalhos futuros, pode-se destacar a utilização de outras medidas a fim de selecionar as interseções em 
regiões de trânsito menos caótico. Além disso, examinar o problema por intermédio de modelos de propagação de sinal ou de obstáculos.

\section{Referências}

Al-Sultan, S., Al-Doori, M. M., Al-Bayatti, A. H., and Zedan, H. (2014). A comprehensive survey on vehicular Ad Hoc network. JNCA, 37(1):380-392.

Aslam, B., Amjad, F., and Zou, C. C. (2012). Optimal roadside units placement in urban areas for vehicular networks. Proceedings - ISCC, 1:423-429.

Ben Brahim, M., Drira, W., and Filali, F. (2015). Roadside units placement within cityscaled area in vehicular ad-hoc networks. ICCVE, pages 1010-1016.

Blondel, V. D., Guillaume, J.-L., Lambiotte, R., and Lefebvre, E. (2008). Fast unfolding of communities in large networks. JSTAT, 10008(10):6.

Brandes, U. (2001). A faster algorithm for betweenness centrality. Journal of Mathematical Sociology, 25:163-177.

Cavalcante, E. S., Aquino, A. L., Pappa, G. L., and a.F. Loureiro, A. (2012). Roadside unit deployment for information dissemination in a VANET. GECCO, page 27.

Chi, J., Do, S., and Park, S. (2016). Traffic flow-based roadside unit allocation strategy for VANET. BigComp, pages 245-250.

Cumbal, R., Palacios, H., and Hincapié, R. (2016). Optimum Deployment of RSU for efficient communications multi-hop from vehicle to infrastructure on VANET. COLCOM.

Fortunato, S. (2010). Community detection in graphs. Physics Reports, 486(3-5):75-174.

Hochbaum, D. S. (1997). Approximation Algorithms for NP-hard Problems. PWS Publishing Co., Boston, MA, USA.

Khuller, S., Moss, A., and (Seffi)Naor, J. (1999). The budgeted maximum coverage problem. Inf. Process. Lett., 70(1):39-45.

Liang, Y., Liu, H., and Rajan, D. (2012). Optimal placement and configuration of roadside units in vehicular networks. IEEE Vehicular Technology Conference.

Meguerdichian, S., Koushanfar, F., Potkonjak, M., and Srivastava, M. B. (2001). Coverage problems in wireless ad-hoc sensor networks. IEEE INFOCOM, 3:1380-1387.

Newman, M. E. J. (2004). Analysis of weighted networks. Physical Review E - Statistical, Nonlinear, and Soft Matter Physics, 70(5 2).

Silva, C. M., Aquino, A. L., and Jr., W. M. (2015). Deployment of roadside units based on partial mobility information. Computer Communications, 60:28-39.

Silva, C. M., Aquino, A. L. L., and Meira, W. (2014). Design of roadside infrastructure for information dissemination in vehicular networks. NOMS.

Trullols, O., Fiore, M., Casetti, C., Chiasserini, C., and Ordinas, J. B. (2010). Planning roadside infrastructure for information dissemination in intelligent transportation systems. Computer Communications, 33(4):432-442.

Xiong, Y., Ma, J., Wang, W., and Tu, D. (2013). RoadGate: Mobility-centric roadside units deployment for vehicular networks. IJDSN, 2013. 